\title{
A REMARK ON CONVERGENCE OF TEST FUNCTIONS
}

\author{
W. F. MOSS \\ (Received 26 June 1973) \\ Communicated by E. Strzelecki
}

In this note it is shown in the most frequently encountered spaces of test functions in the theory of generalized functions that the customary definitions of convergence are equivalent to apparently much weaker definitions. For example, in the space $\mathscr{S}$ the condition of uniform convergence of the functions together with all derivatives (which appears in the definition of convergence) is equivalent to the condition of pointwise convergence of the functions alone. Thus verification of convergence is simplified somewhat.

The proofs of these results depend on the Fourier transform and are entirely elementary. No specific use is made of theorems of compactness in function spaces.

We recall (see for example, Gel'fand and Shilov (1964), Volume 1, pages 16-17) that the space $\mathscr{S}$ consists of all $C_{\infty}\left(R^{n}\right)$ functions $\phi$ such that there exists positive numbers $C_{j, k}$ independent of $x$ but depending on $\phi$ so that

$$
\left|x^{j} D^{k} \phi(x)\right| \leqq C_{j, k}
$$

for all multi-indices $j$ and $k$. A sequence $\left\{\phi_{m}\right\}$ in $\mathscr{S}$ is said to converge to zero in $\mathscr{S}$ if and

(i) there exist constants $C_{j, k}$ independent of $m$ such that $\left|x^{j} \mathrm{D}^{k} \phi_{m}(x)\right| \leqq C_{j, k}$

(ii) for each multi-index $k,\left\{D^{k} \phi_{m}\right\}$ converges to zero uniformly on every compact subset of $R^{n}$. then

It follows trivially from this definition that if $\left\{\phi_{m}\right\}$ converges to zero in $\mathscr{S}$,

(ii) $\left\{\phi_{m}\right\}$ converges pointwise to zero on $R^{n}$.

More interesting is the following converse.

THEOREM 1. If a sequence $\left\{\phi_{m}\right\}$ in $\mathscr{S}$ satisfies (1) and (ii)', then $\left\{\phi_{m}\right\}$ converges to zero in $\mathscr{S}$.

Before proceeding with the proof we recall (again see Gel'fand and Shilov 
(1967), Volume 1, pages 165-166 and Volume 2, pages 123-125) some further information. The Fourier transform $\psi$ of an element $\phi$ in $\mathscr{S}$

$$
\psi(\sigma)=F[\phi](\sigma)=\int_{R^{n}} e^{i \sigma \cdot x} \phi(\mathrm{x}) \mathrm{dx}
$$

provides a one-to-one mapping of $\mathscr{S}$ onto $\mathscr{S}$ with inverse given by

$$
\phi(\mathrm{x}) \equiv F^{-1}[\psi](x)=(2 \pi)^{-n} \int_{R^{n}} e^{-i \sigma^{\cdot} x} \psi(\sigma) d \sigma .
$$

Moreover, $F$ and $F^{-1}$ are continuous mappings in the sense of convergence defined above; i.e. $\phi_{m}=F^{-1}\left[\psi_{m}\right]$ converges to zero in $\mathscr{S}$ if and only if $\psi_{m}=F\left[\phi_{m}\right]$ converges to zero in $\mathscr{S}$.

PROOF OF THEOREM 1. By well-known properties of the Fourier transform the sequence $\left\{\psi_{m}\right\}$ in $\mathscr{S}$ generated by the Fourier transform of $\phi_{m}, \psi_{m}=F\left[\phi_{m}\right]$, satisfies

$$
(-i \sigma)^{k} D^{j} \psi_{m}(\sigma)=\int_{R^{n}} D^{k}\left[(i x)^{j} \phi_{m}(x)\right] e^{i \sigma \cdot x} d x
$$

It follows easily, because of condition (i), that

$$
\left|D^{k}\left[x^{j} \phi_{m}(x)\right]\right| \leqq C_{j, k}^{\prime} /\left(1+|x|^{2 n}\right) \varepsilon L^{1}\left(R^{n}\right),
$$

where the constants $C_{j, k}^{\prime}$ are independent of $m$. Equations (1) and (2) imply that

$$
\left|\sigma^{k} D^{j} \psi_{m}(\sigma)\right| \leqq C_{j, k}^{\prime \prime}
$$

independent of $m$. Thus $\left\{\psi_{m}\right\}$ meets condition (i) for convergence in $\mathscr{S}$.

If $k$ is the zero multi-index, (1) implies that

$$
\left|D^{j} \psi_{m}(\sigma)\right| \leqq \int_{R^{n}}\left|x^{j} \phi_{m}(x)\right| d x,
$$

and (2) implies that

$$
\left|x^{j} \phi_{m}(x)\right| \leqq C_{J}^{\prime} /\left(1+|x|^{2 n}\right) \varepsilon L^{1}(R) .
$$

Condition (ii)' implies that $\left\{x^{j} \phi_{m}(x)\right\}$ converges to zero pointwise on $R^{n}$. Hence the right hand side of (3) tends to zero as $m \rightarrow \infty$ by Lebesgue's theorem of dominated convergence. This insures that $\left\{\psi_{m}\right\}$ meets condition (ii) for convergence to zero in $\mathscr{S}$. And so $\left\{\psi_{m}\right\}$ converges to zero in $\mathscr{S}$. But then $\left\{\phi_{m}\right\}=\left\{F^{-1}\left[\phi_{m}\right]\right\}$ converges to zero in $\mathscr{S}$, establishing the assertion.

REMARK. On observes that the above proof shows that under the given hypotheses, $\left\{D^{k} \phi_{m}(x)\right\}$ converges uniformly to zero on $R^{n}$. A slight modification shows, in fact, that the same is true for $\left\{x^{j} D^{k} \phi_{m}(x)\right\}$. 
An immediate consequence of Theorem 1 is an analogous result for the space $\mathscr{D}$ (see Gel'fand and Shilov (1964), Volume 1, page 2) of all $C^{\infty}\left(R^{n}\right)$ functions each of which vanishes outside some bounded set (which may vary with the particular function). A sequence $\left\{\phi_{m}\right\}$ in $\mathscr{D}$ converges to zero in $\mathscr{D}$ if

(a) there exists a compact set $K \subseteq R^{n}$ outside of which all the $\phi_{m}$ vanish, and

(b) for each multi-index $k,\left\{D^{k} \phi_{m}\right\}$ converges to zero uniformly on $R^{n}$.

From (b) follows

(b) there exists $C_{k}$ independent of $m$ such that $\left|D^{k} \phi_{m}(x)\right| \leqq C_{k}$ and $\left\{\phi_{m}\right\}$ converges to zero pointwise on $R^{n}$. Our converse is as follows.

COROLLARY 1. If a sequence $\left\{\phi_{m}\right\}$ satisfies (a) and (b)', then $\left\{\phi_{m}\right\}$ converges to zero in $\mathscr{D}$.

Proof. If $\phi_{m}(x)$ vanishes identically for $\left|x_{i}\right| \geqq r_{i}$ for $m=1,2, \cdots$, then

$$
\left|x^{j} D^{k} \phi_{m}(x)\right| \leqq r^{j} C_{k} .
$$

Since $\mathscr{D} \subseteq \mathscr{S}$, theorem 1 implies $\left\{\phi_{m}\right\}$ converges to zero in $\mathscr{S}$. Hence $\left\{D^{k} \phi_{m}\right\}$ converges to zero uniformly for $\left|x_{i}\right| \leqq r_{i}$, yielding the assertion.

Another consequence of theorem 1 is an analogous result for the space $\mathscr{Z}$ (see Gel'fand and Shilov (1964) Volume I, pages 155-157) consisting of all entire functions $\psi(s), s=\left(s_{1}, \cdots, s_{n}\right)$ of $n$ complex variables, $s_{i}=\sigma_{i}+i \tau_{i}$, for which there exist constants $C_{q}$ and a vector $r$ in $R^{n}$ with $r_{i}>0$ (independent of $s$ but depending on the particular element $\psi$ of $\mathscr{Z}$ ) such that

$$
\left|s^{q} \psi(s)\right| \leqq C_{q} e^{r_{1}\left|\tau_{1}\right|+\ldots+r_{n}\left|\tau_{n}\right|}
$$

A sequence $\left\{\psi_{m}\right\}$ in $\mathscr{Z}$ converges to zero in $\mathscr{Z}$ if

$(\alpha)$ there exists $C_{q}$ and $r$ independent of $m$ such that

$$
\left|s^{q} \psi_{m}(s)\right| \leqq C_{q} e^{r_{1}\left|t_{1}\right|+\ldots+r_{n}\left|r_{n}\right|} .
$$

and

( $\beta)\left\{\psi_{m}\right\}$ converges uniformly to zero on every compact subset of $R^{n}$. From ( $\beta$ ) follows

$(\beta)^{\prime}\left\{\psi_{m}\right\}$ converges pointwise to zero on $R^{n}$.

Our converse is as follows.

COROLlaRY 2. If a sequence $\left\{\psi_{m}\right\}$ in $\mathscr{Z}$ satisfies $(\alpha)$ and $(\beta)^{\prime}$, then $\left\{\psi_{m}\right\}$ converges to zero in $\mathscr{Z}$.

Proof. We recall (see Gel'fand and Shilov (1964), Volume 1, pages 155-157 and Volume 2, pages 126-127) that the Fourier transform

$$
\psi(s)=F[\phi](\mathrm{s})=\int_{R^{n}} e^{i s^{\cdot} x} \phi(x) d x
$$

provides a one-to-one mapping of $\mathscr{D}$ onto $\mathscr{Z}$ and that $\psi_{m}=F\left[\phi_{m}\right]$ converges 
to zero in $\mathscr{Z}$ if and only if $\phi_{m}=F^{-1}\left[\psi_{m}\right]$ converges to zero in $\mathscr{D}$. Moreover, if $\left\{\psi_{m}\right\}$ satisfies condition ( $\alpha$ ), then $\left\{\phi_{m}\right\}$ fulfills condition (a). Also,

$$
D^{k} \phi_{m}(x)=(2 \pi)^{-n} \int_{R^{n}}(-i \sigma)^{k} e^{i \sigma \cdot x} \psi_{m}(\sigma) d \sigma .
$$

Equation (4) and condition ( $\alpha$ ) imply that

$$
\left|D^{k} \phi_{m}(x)\right| \leqq C_{k} .
$$

Equation (4) with $k$ the zero multi-index, together with conditions $(\alpha)$ and $(\beta)^{\prime}$, insure the pointwise convergence of $\left\{\phi_{m}\right\}$ to zero by Lebesque's theorem of dominated convergence (as in the proof of theorem 1). Thus $\left\{\phi_{m}\right\}$ meets conditions (a) and (b)' and so, by corollary 1 , converges to zero in $\mathscr{D}$. Consequently, $\left\{\phi_{m}\right\}$ converges to zero in $\mathscr{Z}$ by the continuity of $F$.

\section{Reference}

I. M. Gel'fand and G. E. Shilov (1964), Generalized Functions (Academic Press, New York, 1964).

University of Delaware

Newark Delaware

U. S. A. 